\title{
Paraneoplastic ophthalmoplegia and subacute motor axonal neuropathy associated with anti-GQ1b antibodies in a patient with malignant melanoma
}

\author{
L Kloos, P Sillevis Smitt, C W Ang, W Kruit, G Stoter
}

J Neurol Neurosurg Psychiatry 2003;74:507-509

A 68 year old woman developed oculomotor paresis shortly after metastatic progression of her melanoma was discovered. She was then immunised with the tumour antigen MAGE-3 in combination with an immunological adjuvant. During immunisation her symptoms worsened and she developed severe, predominantly proximal axonal motor neuropathy and became bedridden. IgM antibodies against gangliosides GM2, GD3, and GQ1b were detected in serum obtained two weeks before and nine weeks after the onset of symptoms. Immunohistochemically, the patient's IgM reacted with the tumour and co-localised with GQ1b. She improved neurologically following steroid treatment and became ambulatory.

M alignant melanoma is a potentially immunogenic tumour, and the development of melanoma vaccines has been an important line of research. Tumour specific antigens used as targets of melanoma immunotherapy are often surface glycoproteins that share immunogenic similarities with glycoproteins on the surface of normal melanocytes and cells in the central and peripheral nervous systems. The immune response against these melanoma antigens may also cross react with normal melanocytes or neurones, resulting in several clinical symptoms such as vitiligo, inappropriate secretion of antidiuretic hormone (SIADH), and chronic inflammatory demyelinating polyneuropathy (CIDP). We describe a patient with malignant melanoma who developed oculomotor paresis followed by a subacute motor axonal neuropathy associated with antiganglioside antibodies suggesting cross reactivity ("molecular mimicry") between melanoma and peripheral nerve antigens.

\section{CASE REPORT}

The patient had been diagnosed with a malignant melanoma on her left foot in 1983. This was surgically excised (Breslow thickness $1.9 \mathrm{~mm}$ ). In March 2000, at the age of 68, she complained of night sweating and weight loss of $17 \mathrm{~kg}$ over the previous six months. Computed tomography (CT) showed extensive retroperitoneal and left iliac lymphadenopathy. A CT guided biopsy of an iliac lymph node was undertaken and pathological examination showed malignant melanoma. Reverse transcriptase polymerase chain reaction (RT-PCR) showed tumour expression of the tumour antigen MAGE-3, and the patient was included in an immunotherapy trial. The treatment consisted of three intramuscular vaccinations at weekly intervals with $300 \mu \mathrm{g}$ MAGE-3 recombinant protein in combination with immunological adjuvant.

In June, one week before the first vaccination, she developed double vision. Two weeks later she was referred to the neurology clinic. Examination revealed fluctuating exter- nal ophthalmoplegia. Motor and sensory examination was normal apart from absent deep tendon reflexes.

One month later (five weeks after the first vaccination), she developed rapidly progressive and predominantly proximal motor weakness: MRC muscle strength grade 3 in the deltoids and hip flexors, grade 4 in biceps and triceps, and grade 5 distally; weakness in the neck flexors was MRC grade 2 . Sensory examination remained normal. The patient was admitted to our hospital for further investigation.

Cranial and spinal magnetic resonance imaging (MRI) before and after gadolineum administration was normal. The CSF was acellular with raised protein $(1.9 \mathrm{~g} / \mathrm{l})$ and repeatedly negative cytology (on six occasions). Other laboratory studies were unremarkable including creatine kinase, calcium, and thyroid function studies. The erythrocyte sedimentation rate was $68 \mathrm{~mm} /$ hour. Antibodies to acetylcholine receptor and voltage gated calcium channels were negative and no antibodies were detected against the paraneoplastic $\mathrm{Hu}, \mathrm{CV}-2$, Ri, Yo, or amphiphysin antigens. A neostigmine provocation test produced no improvement in her symptoms. Electrophysiological studies showed normal motor and sensory nerve conduction velocities with normal or only mildly decreased motor amplitudes. There were no signs of dispersion or conduction block. Needle electromyography showed widespread acute denervation changes in the proximal muscles and mild denervation in the distal muscles. Low frequency $(3 \mathrm{~Hz})$ and high frequency $(20 \mathrm{~Hz})$ repetitive nerve stimulation did not change the compound muscle action potentials. Because of the proximal distribution of the weakness, a deltoid muscle biopsy was taken. Pathological examination did not show myelitis or myopathy. Sural nerve biopsy showed signs of axonal degeneration without inflammatory cells or immunoglobulin deposits.

The MAGE-3 vaccinations were stopped and she was treated with intravenous immunoglobulin $(0.4 \mathrm{~g} / \mathrm{kg} / \mathrm{d}$ for five days). Despite this, her weakness deteriorated and she developed dysphagia and dysarthria. Subsequent steroid treatment (20 mg dexamethasone daily) resulted in a remarkable improvement in strength and bulbar function within two days. She was discharged from hospital on $12 \mathrm{mg}$ dexamethasone a day with a gradual taper. There was continuing improvement in her symptoms and after six weeks she was able to walk again without help. Because of relapsing diplopia she was treated with five plasma exchanges, resulting in subjective improvement. She has remained steroid dependent (dexamethasone $6 \mathrm{mg} / \mathrm{d}$ ).

\section{METHODS}

Serum samples were obtained from the patient two weeks before the onset of symptoms (three weeks before the vaccination was started) and nine weeks after the onset of symptoms (eight weeks after the vaccination). Both samples were analysed for the presence of antiganglioside antibodies against GM1, asialo-GM1, GM2, GM3, GDla, GDlb, GD3, 


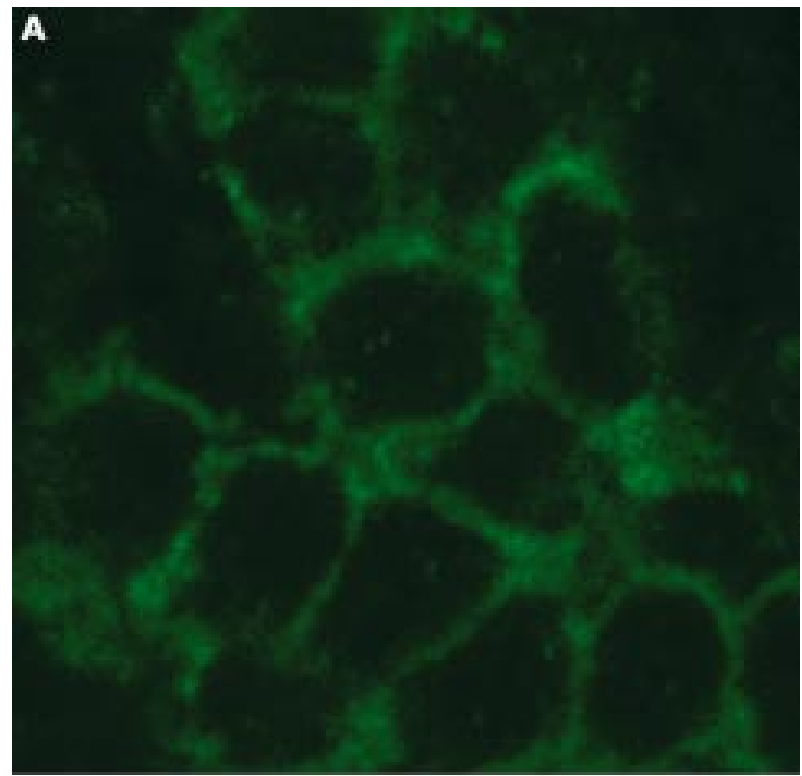

B

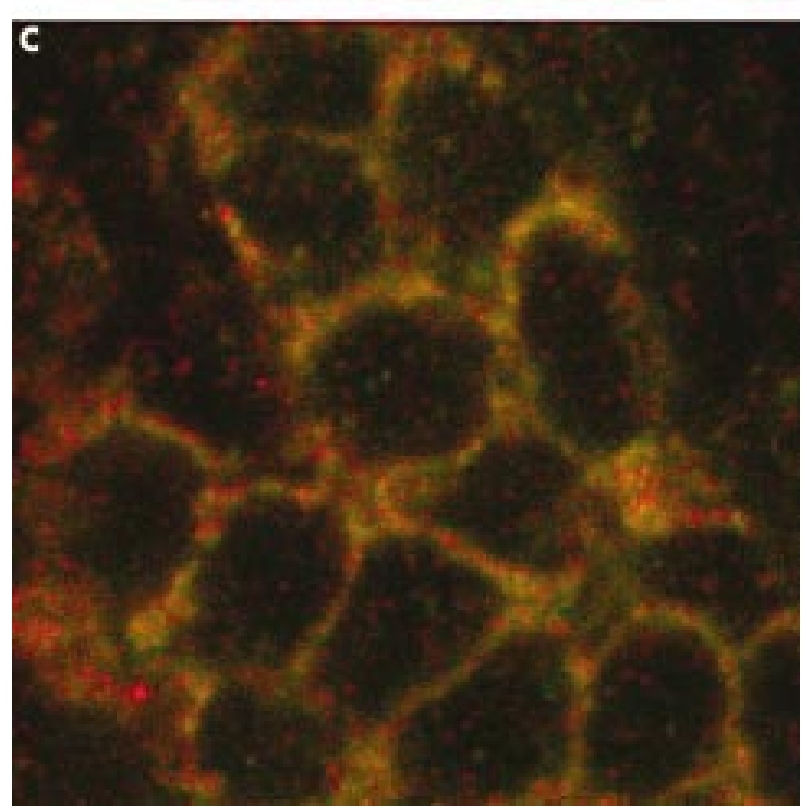

GTIb, and GQIb with an enzyme linked immunosorbent assay (ELISA) and confirmed with thin layer chromatography as described before. ${ }^{1}$ Serum was also tested for the presence of IgM and IgA antibodies against Campylobacter jejuni, cytomegalovirus, Epstein-Barr virus, and Mycoplasma pneumoniae, as described. ${ }^{2}$ Indirect immunofluorescent examination was undertaken on formalin fixed, paraffin embedded sections of the primary melanoma and the metastasis. ${ }^{3}$ In brief, the sections were prepared for double immunofluorescent labelling by microwave preparation (five minutes at $900 \mathrm{~W}$ ) in citric acid ( $\mathrm{pH}$ 6) followed by five minutes of $0.1 \%$ pronase treatment at $37^{\circ} \mathrm{C}$. The patient's serum was diluted 1:200 and polyclonal rabbit anti-GQ1b, R2327E diluted 1:100 was added, followed by rhodamine and fluorescein labelled secondary antibodies. Antibody specificity was determined by incubation with a non-immune serum and by leaving out the primary serum. Images were analysed and photographed using a confocal fluorescent microscope.

\section{RESULTS}

Thin layer chromatographic overlay of the patient's serum to multiple purified gangliosides revealed strong and specific binding of IgM with GM2 (titre 200), GQ1b (titre 400), and GD3 (titre 200). The patient's serum did not react with the gangliosides asialo-GMl, GMl, GM3, GDla, and GTlb. Weak background reactivity with GDlb was observed. The titres of the pre- and postvaccination samples (obtained two and nine weeks after the onset of symptoms, respectively) were the same. There was no IgG reactivity with any of the gangliosides tested. The patient's serum did not contain monoclonal bands as determined by immunoelectrophoresis. No evidence for recent infection with Campylobacter jejuni, cytomegalovirus, Epstein-Barr virus, or Mycoplasma pneumoniae could be detected.

On indirect immunofluorescence, the patient's IgM reacted with many of the tumour cells, as shown in fig lA (fluorescein filter). Antiserum to GQIb reacted with many of the same cells (fig 1B), as visualised with the rhodamine filter. Colocalisation is confirmed with double exposure in yellow (fig IC).

\section{DISCUSSION}

The patient presented with external ophthalmoplegia in combination with areflexia and a high CSF protein concentration, with a normal CSF cell count and anti-GQlb antibodies. She subsequently developed a severe subacute motor axonal neuropathy which made her bedridden within two months and was accompanied by involvement of the lower cranial nerves.

The patient's neuropathy developed shortly after the diagnosis of recurrent melanoma and before vaccination, suggesting a paraneoplastic aetiology. Neither ophthalmoplegia nor subacute motor axonal neuropathy has previously been reported as paraneoplastic syndromes associated with melanoma. ${ }^{5}$ In this patient, other cancer related causes such as leptomeningeal metastases and direct invasion of the peripheral nerves by the tumour were excluded.

Most paraneoplastic neurological syndromes are considered autoimmune disorders caused by an immune response directed against antigens in the tumour which subsequently (cross) react with the same or similar epitopes in the nervous system. The patient had IgM autoantibodies in her serum which reacted with the gangliosides GM2, GD3, and GQ1b. All three antigens are immunogenic and are expressed on

Figure 1 The patient's $\lg M$ immunoreacts with many of the tumour cells visualised in green with fluorescein filters (panel A). Anti-GQ1b antiserum co-localises with most of the same cells as shown in red with rhodamine filters (panel B). Co-localisation is confirmed in yellow (panel C) by double exposure from the superimposition of red and green. 
melanoma tumours and cell lines, suggesting that the tumour may have triggered the production of antibodies. Indeed, the patient's IgM also reacted with the melanoma tissue, co-localising with GQlb. An immune aetiology of the neuropathy is further suggested by the remarkable, albeit partial, response to steroid treatment.

The importance of each of the target glycolipids for the pathophysiological mechanism remains unclear. Anti-GQlb reactivity is strongly associated with oculomotor symptoms in patients with immune mediated neuropathies. ${ }^{6}$ Moreover, in $20 \%$ of patients with Guillain-Barré syndrome and anti-GQlb antibodies, the EMG changes are mainly axonal, as in our patient. ${ }^{7}$ In vitro models have clearly shown that anti-GQlb antibodies disrupt the neuromuscular junction, resulting in breakdown of axonal terminals; this suggests that they can be pathogenic in vivo. ${ }^{8}$ Anti-GM2 IgM antibodies can also affect neuromuscular transmission. ${ }^{9}$ A pathogenic role of these antibodies in our patient is less likely, because anti-GM2 antibodies are associated with demyelinating neuropathies and their specificity is questioned. Anti-GD3 antibodies most probably result from cross reactive epitopes with GQlb.

Other forms of neuropathy have been described in association with melanoma, including five reported cases of chronic inflammatory demyelinating polyneuropathy (CIDP). ${ }^{310}$ A further pathophysiological relation between melanoma and neuropathy is suggested by reports of demyelinating neuropathy following vaccination with melanoma lysates. ${ }^{11}$ In contrast to the previously reported cases, the neuropathy in our patient was strictly motor, axonal, and accompanied by upper and lower cranial nerve involvement.

The relation between the vaccinations and worsening of the neuropathy remains unclear. A direct relation with immunity directed against MAGE-3 is highly unlikely because MAGE-3 is a tumour specific antigen that is not expressed in the nervous system. ${ }^{12}$ Furthermore, the neuropathy and antiganglioside antibodies were clearly present before vaccination, and the titres of the antibodies were not influenced by the vaccinations. It is difficult to conclude whether the evolution of symptoms was spontaneous, or whether concomitant vaccination played an indirect role. Clearly, stimulation of the immune system-whether through an infectious process or through vaccination-could theoretically promote autoimmune phenomena by causing a systemic increase of inflammatory cytokines. Although there is no evidence that MAGE-3 vaccination caused the worsening of the neuropathy, our observation suggests the need for caution when inducing immune responses in patients with ongoing autoimmune symptoms.

\section{ACKNOWLEDGEMENTS}

We gratefully acknowledge technical assistance by Esther Hulsenboom, Hans Vuik, and Adriaan Houtsmuller.

\section{Authors' affiliations}

L Kloos, P Sillevis Smitt, Department of Neurology, Erasmus University Medical Centre, Rotterdam, Netherlands

C W Ang, Department of Medical Microbiology and Infectious Diseases, Erasmus University Medical Centre

W Kruit, G Stoter, Department of Medical Oncology, Erasmus University Medical Centre

L Kloos is presently at the Neurology Department, Leyenburg Ziekenhuis, Den Haag, The Netherlands

Competing interests: none declared

Correspondence to: Dr Peter Sillevis Smitt, Department of Neurology, Erasmus University Medical Centre, Dr Molewaterplein 40, 3015 GD Rotterdam, Netherlands; sillevis@neuh.azr.n

Received 20 October 2002

Accepted 2 December 2002

\section{REFERENCES}

1 Ang CW, Endtz HP, Jacobs BC, et al. Campylobacter jejuni lipopolysaccharides from Guillain-Barré syndrome patients induce lgG anti-GM1 antibodies in rabbits [in process citation]. J Neuroimmunol 2000;104:133-8.

2 Jacobs BC, Rothbarth PH, van der Meche FG, et al. The spectrum of antecedent infections in Guillain-Barré syndrome: a case-control study. Neurology 1998;51:1110-15.

3 Weiss MD, Luciano CA, Semino-Mora C, et al. Molecular mimicry in chronic inflammatory demyelinating polyneuropathy and melanoma. Neurology 1998;51:1738-41.

4 Ang CW, De Klerk MA, Endtz HP, et al. Guillain-Barré syndrome- and Miller Fisher syndrome-associated Campylobacter jejuni lipopolysaccharides induce anti-GM1 and anti-GQ1b antibodies in lipopolysaccharides induce anti-GMI and

5 Sillevis Smitt PAE, Posner JP. Paraneoplastic peripheral neuropathy. In: Hartung H-P, ed. Peripheral neuropathies, part I. London: Baillière Tindall, 1995:443-69.

6 Odaka M, Yuki N, Hirata K. Anti-GQlb lgG antibody syndrome: clinical and immunological range. J Neurol Neurosurg Psychiatry $2001 ; 70: 50-5$

7 Jacobs BC, Meulstee J, van Doorn PA, et al. Electrodiagnostic findings related to anti-GM1 and anti-GQ1b antibodies in Guillain-Barré syndrome. Muscle Nerve 1997;20:446-52.

8 O'Hanlon GM, Plomp JJ, Chakrabarti M, et al. Anti-GQ1b ganglioside antibodies mediate complement-dependent destruction of the motor nerve terminal. Brain 2001;124:893-906.

9 Ortiz N, Rosa R, Gallardo E, et al. IgM monoclonal antibody against terminal moiety of GM2, GalNAc-GD 1 a and GalNAc-GM1b from a pure motor chronic demyelinating polyneuropathy patient: effects on neurotransmitter release. J Neuroimmunol 2001;119:114-23.

10 Anthoney DA, Bone I, Evans TR. Inflammatory demyelinating polyneuropathy: a complication of immunotherapy in malignant melanoma. Ann Oncol 2000;1 1:1 197-200.

11 Fuller GN, Spies JM, Pollard JD, et al. Demyelinating neuropathies triggered by melanoma immunotherapy. Neurology 1994;44:2404-5.

12 van der Bruggen $\mathbf{P}$, Traversari $C$, Chomez $P$, et al. A gene encoding an antigen recognized by cytolytic $T$ lymphocytes on a human melanoma. Science 1991;254:1643-7. 


\section{CORRESPONDENCE}

\section{The naming of parts}

Many deplore the journalistic trend to label well recognised conditions by acronyms, or by recently invented names-commonly to no useful purpose. Thus neurologists may not welcome yet another two names, recorded in past literature but not in general currency.

Umapathi et al give a most valuable and timely review of the dropped head and bent spine syndromes. ${ }^{1}$ But why perpetuate the ungainly phrase head ptosis? Ptosis, Greek $\pi \tau \omega \sigma \mathrm{s} \varsigma=$ falling, has traditionally been applied only to the upper eyelid and to prolapse of any of the viscera or of the breasts. Head drop is short and its meaning is unequivocal.

Camptocormia, Greek $\kappa \alpha \mu \pi v \lambda=$ bent $\kappa о \rho \mu о \varsigma=$ trunk of a tree: but, like a few others, ${ }^{2}$ Umapathi et al apply it to signify a bent spine. Camptocormia was first an illnes occurring among soldiers in World Wars I and II, and was regarded as a sign of hysteria. Ankylosing spondylitis is a more frequen cause. It is an ostentatious word that portends something more mysterious than a bent back or neck. To be similarly pretentious, can we not survive without such euphuisms?

J M S Pearce Anlaby, East Yorks, UK; imsp@freenet.co.uk

\section{References}

1 Umapathi T, Choudhry V, Cornblath D, et al. Head drop and camptocormia. I Neuro Neurosurg Psychiatry 2002;73:1-8.

2 Sinel M, Eisenberg MS. Two unusual gait disturbances: astasia, abasia and camptocormia. Arch Phys Med Rehabil 1990;71:1078-80.

\section{Head drop and camptocormio}

The article by Umapathi $e t$ al ${ }^{1}$ in this journal referred to the original use of the term camptocormia by Souques in 1915, though functional bent back was first described by Brodie in 1837. Mlle Rosanoff-Saloff supported Souques' case study with a photographic record of this soldier's bent back and his recovery. According to the English translation abstract in Southard's fine collection of shell shock cases $^{3}$ this soldier was wounded five month previously by a bullet that entered along the auxiliary border of the scapula and emerged near the spine. "He spat blood for several days ... and when he got up his trunk and thighs were found to be in a state of moderate flexion upon the pelvis, the trunk being bent almost at a right angle." He was able to bend his trunk still further forward than its habitual contractured position' and it was evident that there was contraction of the muscles of the abdominal wall and of the iliopsoas. "No motor, sensory, reflex, trophic, vasomotor, electrical, visceral or X-ray disorders could be found." The application of plas ter corsets 'cured' this man's deformity within six weeks.

The poilus spoke of this condition as cintrage (arching), suggesting that it was not an uncommon affliction of the French soldier. Seemingly only recorded by French neurologists, Roussy and Lhermitte reported two subsequent cases. ${ }^{3}$ An infantryman was thrown into the air by the bursting of a shell, rendered unconscious and recovered experiencing violent pains in the back. He remained stooped to the right. His bent back was corrected by the application of plaster corsets. The other reported case was that of a chasseur who was buried in an explosion, knocked unconscious, and experienced acute respiratory distress, and subsequent mutism and camptocormia. One séance of electrical treatment corrected the improper attitude of the trunk, though he did continue to experience "a few persistent lumbar pains".

It would be difficult to doubt the probability that psychological factors influenced these men's recuperation. To describe these soldiers as hysterical, ${ }^{1}$ though this was the terminology used during this period, or indeed that they suffered functional bent back, is probably unfair. They may well have suffered acute traumatic spinal injury and reactive muscle spasm (and contractures). Persistent stooping in shallow trenches, in appalling conditions of deprivation and danger, may have been contributing factors weakening the tone of paraspinal muscles. However, these case reports suggest that the traumatic injury alone may be sufficient explanation for the bent spines. The management of camptocormia in the first world war was to provide biomechanical supports, such as corsets, apparently with good results. The psychological therapies of "persuasive reeducation" were additive rather than pivotal and faradisation (and other tortures) used only "if necessary". ${ }^{3}$

The Sandler triad of low self esteem with confusion of identity, sadomasochistic behaviour toward military authorities, and impotence ${ }^{4}$ were, in 1947, proposed as being an essential part of camptocormia. Umapathi's ${ }^{1}$ recognised causes of camptocormia and the contributing factors however implicate organicity, as indeed do the original case reports.

A D Macleod Psychiatric Consultation Service, Christchurch Hospital, Private Bag 4710, Christchurch, New Zealand; PCS@cdhb.govt.nz

\section{References}

1 Umapathi T, Chaudhry V, Cornblath D, et al. Head drop and camptocormia. J Neurol Neurosurg Psychiatry 2002;73: 1-7.

2 Souques A, Rosanoff-Saloff M. La camptocormie. Rev Neurol (Paris) 1914;22:937-9.

3 Southard EE. Shell-shock and other neuropsychiatric problems presented in 589 case histories from the war literature, 1914-1918. Boston: WM Leonard, 1919

4 Sandler SA. Camptocormia, or the functional bent back. Psychosom Med

1947;9: 197-204.

\section{Author's reply}

We would like to thank Dr J M S Pearce for his comments.

We agree with him on the proliferation of medical terms referring to similar if not identical conditions. One of the chief aims of writing this paper is to thread a line of commonality through the various names in literature, which in essence refer to an anterior curvature of the spine. Hence the title "Head drop and camptocormia, the spectrum of bentspine disorders".

However, we would like to disagree with Dr Pearce labelling the spinal deformity seen in ankylosing spondylitis as camptocormia. In arthritic conditions and diseases that affect bone, the spinal deformity is fixed. In the bent-spine disorders referred to in the paper, the deformity may reduce considerably or even disappear with change in position, for example when supine. We would therefore prefer to reserve the phrases head drop (used interchangeably with head ptosis) and camptocormia to neurological conditions that affect the strength or tone of the muscles controlling spinal posture.

As aficionados of medical history, we very much enjoy Dr A D Macleod's letter. We agree that organic factors might have contributed to the camptocormia in solders believed to have been suffering from hysteria. It would have not been unexpected for patients, like the man described by Southard with a bullet wound near the spine, ${ }^{1}$ to have developed spasm or even denervation of thoracic paraspinal muscles.

T Umapathi

Department of Neurology, National Neuroscience Institute, 11 Jalan Tan Tock Seng, 308433 Singapore; tumapathi@yahoo.com

\section{Reference}

1 Southard EE. Shell shock and other neuropsychiatric problems presented in 589 case histories from the war literature 1914-1918. Boston: WM Leonard, 1919.

\section{Infection and multiple sclerosis}

The article by Hawkes ${ }^{1}$ and the editorial commentary about the role of infectious agents in multiple sclerosis (MS) examined this question from a new viewpoint based on epidemiological observations. ${ }^{2}$ Several infectious agents, most not sexually transmitted, were reported to be associated with MS according to epidemiological data, serology in CSF and blood, or demonstration of pathogens in tissue. A relation with measles virus (MV) has been an early and most consistent finding. More recently, higher prevalence and higher titres of antibodies against human herpesvirus 6 (HHV6), but not other herpesviruses, were shown in MS patients compared to control groups, suggesting different exposure to HHV6 in MS. ${ }^{3}$ HHV6, like vaccine strain $M V$ and certain wild type $M V$, uses the membrane cofactor protein (MCP; CD46) as a receptor for entry into cells. This suggests a possible involvement of CD46 in MS.

The possibility of a particular isoform of CD46 predisposing MS patients to infection is unlikely because all isoforms have similar affinity to MV. Increased levels of soluble CD46 have been reported in the serum and cerebrospinal fluid of MS patients, more in those who have HHV6 DNA. ${ }^{4}$ One interpretation of these findings involved increased activity of the complement system in MS. However, experimental studies show no influence of inflammatory cytokines on CD46 expression and do not support inflammation 
as a cause of increased CD46. Incorporation of CD46 in the viral envelope, or a possible genetic propensity in MS patients, have also been considered as causes of increased CD46. ${ }^{4}$ While its origin in MS is unclear, soluble CD46 might be involved in viral pathogenesis by binding the virus in the viraemic phase and allowing another to attach to CD46 and spread from cell to cell. Both HHV6 and MV are infectious agents encountered in early childhood, and HHV6 can indeed become reactivated a few weeks after primary MV infection. On the other hand, because HHV6 and MV downregulate CD46 expression on the infected cell, they may diminish the entry of each other, delaying the time of infection. Therefore, they might produce increased antibody levels in young adults through delayed infection with, or reactivation of, each other. These suggest increased antibodies against these two viruses in MS may be interrelated.

The question remains whether a causeeffect relation exists between infectious organisms and MS, or whether viruses are just a consequence of the activation of the inflammatory-immune sequence or increased susceptibility of MS patients to infection. Studies of CD46 and other viral receptors seem warranted in MS.

B Anlar

Department of Pediatric Neurology, Hacettepe University, Ankara 06100, Turkey; banlar@hacettepe.edu.tr

\section{References}

1 Hawkes $\mathrm{CH}$. Is multiple sclerosis a sexually transmitted infection? J Neurol Neurosurg Psychiatry 2002;73:439-43.

2 Stewart G. Infection and multiple sclerosis-a new hypothesis? J Neurol Neurosurg Psychiatry 2002;73:358-9.

3 Enbom M. Human herpesvirus 6 in the pathogenesis of multiple sclerosis. APMIS 2001;109:401-11.

4 Soldan SS, Fogdell-Hahn A, Brennan MB, et al. Elevated serum and cerebrospinal fluid levels of soluble human herpesvirus type 6 cellular receptor, membrane cofactor protein, in patients with multiple sclerosis. Ann Neurol 2001;50:486-93.

5 Mason JC, Yarwood H, Sugars K, et al. Induction of decay-accelerating factor by cytokines or the membrane-attack complex protects vascular endothelial cells against complement deposition. Blood 1999;94:1673-32.

\section{Infection and multiple sclerosis}

The paper by $\mathrm{C} \mathrm{H}$ Hawkes (Is multiple sclerosis a sexually transmitted infection? $\left.{ }^{1}\right)$ has caused predictable distress to people with multiple sclerosis (MS) and their families. Living with MS is a difficult enough experience without such sudden and avoidable alarm. The UK Multiple Sclerosis Society's national helpline and local branches have been inundated with calls expressing anger and anxiety.

It is hard to understand the motive for publication when your own expert editorial commentator specifically referred to the paper's "pure speculation" and "potential to cause harm". Did the sensational nature of Dr Hawkes's hypothesis and the virtual guarantee of extensive publicity it could receive outweigh proper consideration of its scientific merit?

There is also the worrying question of what damage may have been caused to the reputation of MS research in the UK by the lay media coverage which was attracted. The MS
Society has a current forward commitment of around $£ 12$ million to nearly 70 research projects. That money is raised by voluntary donation. Anything which could discredit the quality of research here is of material concern to us.

M O'Donovan

Chief Executive, The Multiple Sclerosis Society, M S National Centre, 372 Edgware Road, London NW2 6ND, UK; modonovan@mssociety.org.uk

\section{Reference}

1 Hawkes $\mathrm{CH}$. Is multiple sclerosis a sexually

transmitted infection? J Neurol Neurosurg

Psychiatry 2002;73:439-43.

Ed: The journal regrets any distress caused to patients with MS as a result of the widespread publicity this article received in the media.

However, we wish to emphasise that the article was subject to the usual peer review process.

\section{BOOK REVIEWS}

\section{Delirium in old age}

Edited by J Lindesay, K Rockwood, A Macdonald (Pp 238, £55.00). Published by Oxford University Press, Oxford, 2002. ISBN 0-19-263275-2

Delirium is an extremely important condition for a number of reasons. It is very distressing and frightening for those who experience the symptoms, and descriptions of the effects on the brain as a result of high fever have been well described. There is a high mortality associated with the development of delirium, and it is often associated with behavioural disturbances that can be troublesome for carers and attendants. Finally, it presents a unique opportunity to look at the interface between psychiatric symptoms caused by organic disease and functional disorders.

Twelve years ago, the same publishers and two of the current editors produced the first edition on delirium. It was a relatively thin book but set the standards that the current edition continue. Delirium is certainly a niche market, and there appear to be no direct competitors, although textbooks on old age psychiatry usually contain chapters and notes on delirium. The new edition is greatly expanded and very much up to date.

Every aspect of delirium is included, from the history and conceptual basis of the disorder through epidemiology, neurophysiology, clinical assessment, management, prevention, and, refreshingly, the the role of family caregivers and nurses in managing the disease. The core tenet of the book is that delirium is a disorder that is relatively poorly recognised (particularly the hypo-alert type) by the general clinical professions, it is relatively easy to identify people at risk of developing delirium, and that there is a real possibility of a reasonable preventive strategy for the disorder. Twelve authors have contributed and, as delirium is relatively under-researched, this probably represents a significant proportion of the leading researchers in the field internationally. There are particularly interesting sections on the conceptual basis of the disorder and how it, and its component symptoms, are defined, methods of assessment of delirium are covered comprehensively, a summary of how evidence based management plans can be developed, and the prospects of prevention of delirium are given an adequate airing.

An interesting spin, which I discovered by accident, is that on the Oxford University Press website (www.oup.co.uk), one can see online updates of each individual chapter. Those present when this author last visited the website (December 2002) consisted of work that had been done from when the manuscript had been submitted to publication. It may be that reviews of the book might also appear online-this one will.

The book is a landmark in the literature on delirium, is a text of very high quality, and anyone seriously involved in the clinical management of patients with delirium or research on the subject would do very well to read this book.

A Burns

\section{Neurophysiology in neurosurgery. A modern intraoperative approach}

Edited by Vedran Deletis and Jay L Shils (Pp 469, \$125.00). Published by Academic Press, California, 2002. ISBN 0-12209036-5

This book comprises 17 chapters contributed by 24 authors. It has clearly benefited from most of the chapters being written in a more or less homogenous style and formed into seven parts based mainly on surgical procedures: motor evoked potentials/ neurophysiological base; intraoperative neurophysiology (ION) of the spinal (spinal cord monitoring); ION of peripheral nerves, nerve roots and plexuses; ION of cranial nerve and brainstem; ION of supratentorial procedures; ION during stereotactic neurosurgery for movement disorders; and ION and anaesthesia management. Most of the chapters cover the background of methodology, description of the surgical procedure, and the related neurophysiological procedure, personal experience, and case reports, which gives a balanced theoretical and practical view on the topic of each chapter. The interdisciplinary approach taken in this book will ensure it has a wide range of readers across "neurosurgery, neurology, orthopaedic surgery, neurophysiology, anesthesiology, interventional radiology, and biomedical engineering"

Chronic deep brain stimulation or neuromodulation has extended the role of clinical neurophysiology beyond its traditional diagnostic role. This new field is touched upon briefly in the part on ION during stereotactic neurosurgery. An interesting feature of this book is that it is accompanied by a CD that certainly enhances its value. Cross references are given at the end of the corresponding chapter rather than in the list of contents in the book, and at the front page of the display.

In conclusion, it is an authoritative review of intraoperative neurophysiology much weighted on the motor system for a wide range of surgical procedures. Perhaps, in its present form, those hoping for a more systematically informed discussion on intraoperative neurophysiology of the sensory system may feel slightly disappointed.

X Liu, T Z Aziz 


\section{Clinical neurophysiology of the vestibular system, 3rd edition}

Edited by R.W. Baloh and V. Honrubia (Pp 408, US\$98.50). Published by Oxford University Press, New York, 2001. ISBN 0-19513982-8.

The first edition of Clinical neurophysiology of the vestibular system, published in 1979, had a significance beyond its content: it affirmed that neurology had a stake in the vestibular system. Here was a neurologist (Baloh) writing with an otolaryngologist (Honrubia) about semicircular canals, endolymph, audiograms, and above all the vestibulo-ocular reflex-the "VOR". The VOR is no ordinary reflex; one can measure accurately both its input and its output and come up with a transfer function for gain-a new concept then for neurology. We have learnt a lot more about measurement of vestibular function and about disorders of the vestibular system since 1979. The 2nd edition, published in 1990, and now the third edition, incorporate these advances.

And what a terrific book it still is: based on concepts, packed with facts, lucidly written, and rigorously referenced. Its structure is logical and its language is clear, so that it is not only easy to search and browse but a pleasure to read from cover to cover. And it is comprehensive-no vestibular stone is left unturned.

There are four main parts, dealing in turn with: the structure and function of the vestibular system (four chapters); the clinical and laboratory evaluation of the dizzy patient (four chapters); specific diseases affecting the vestibular system (10 chapters); and the treatment of vertigo and vestibular loss (two, yes only two, chapters-but then that's neurology for you).

It's impossible to single out any one chapter they are all outstanding. For example, I particularly liked the new material in chapter one on the phylogeny of the vestibular system. Now one would have to admit that familiarity with the otocyst of the sea anemone is not a lot of use in the consulting room, but this section is so clearly written and matter so interestingly explained that one happily dispenses with such utilitarian demands.

The great strength of the book and what has made it such a classic, is that although it is based on physiology, full comprehension of physiology is not a prerequisite for retrieving useful information from the disease based chapters. Although the structure is there, one can put this aside and simply delve. The chapters on the three most common vestibular diseases, benign positional vertigo, migraine, and Meniere's diseases, are absolute gems. Each could be published as a self-contained review in its own right.

The book is an elegant conceptual and factual account of the vestibular system, its disorders and diseases, rather than a self-help or how I do it manual. Some readers might miss not having, a "frequently asked clinical questions" section, or at least a "frequently encountered clinical pitfalls" section, but then no one can have it all. Anyone who sees dizzy patients needs one dizzy book on the desk. This is the one I have on mine.

G M Halmagyi

\section{Role of proteases in the pathophysiology of neurodegenerative diseases}

Edited by Abel Lajtha and Maren L Banik (Pp 302 , £59.50). Published by Kluwer 2001. ISBN 0-306-46579-5

This volume would be an extremely useful addition to the bookshelf of anybody with an active interest in the biochemical and pathological processes that underlie some of the more common neurological diseases. In the past the role of proteolysis in these disorders has been largely neglected because it was assumed that it represented a general nonspecific metabolic process. In terms of attracting research interest the field also suffered from the confusion in the literature concerning the naming of these enzymes and the fact that the same enzyme might have many different names. However, as the editors point out in their preface, this is no longer the case and they have managed to bring together an impressive array of current research on the involvement of proteases in a wide variety of disorders. From what individually might have been regarded as rather disparate studies, one can now start to see common themes not least of which is the potential therapeutic value of targeting specific proteases and the development of specific inhibitors.

If, like me, you don't have specialist knowledge of this area I would recommend going straight to the last chapter on the mammalian proteinase genes. Here you will find a clearly laid out summary of the classification and characteristics of the four main groups of proteases (serine, cysteine, aspartic, and metalloproteinases). I also found the chapter on the ubiquitin/proteasome system and the normal physiological breakdown of proteins particularly informative. Having read these two chapters you then have a wide choice of disorders and proteases to choose from. Perhaps the most widely discussed is Alzheimer's disease, undoubtedly because of the huge advances that have been made in the understanding of the biochemical processes underlying this disease over the past 15 years. Papain-like cysteine proteases (cathepsins), caspases, calpains, and a novel metalloendopeptidase (EC 3.4.24.15) all appear to have some role in the pathology of Alzheimer's disease and may, therefore, be potential targets for drug development. There is also a group of Alzheimer's disease specific proteases that affect the processing of the amyloid precursor protein $(\alpha, \beta$, and, $\gamma$ secretase) and presenilin (presenilinase). Both of these proteins are central to the development of pathology and so these enzymes in particular are key targets for current drug company research.

Apart from the interest in Alzheimer's disease, there are other chapters covering the role of matrix metalloproteinases and calpain in the demyelination of multiple sclerosis and the key role of calpain in the pathology of traumatic brain and spinal cord injury. Further chapters describe the loss of calcium homeostasis and the subsequent pathological activation of calpain, resulting in the breakdown of key structural proteins in some neuromuscular disorders. In summary, this book has something for everyone in an area of research that holds huge promise for the future in terms of developing useful therapies for treating neurodegenerative disorders.

S Gentleman

\section{CORRECTIONS}

The following abstract was not printed with the article by E L J Hoogervorst, M J Eikelenboom, B M J Uitdehaag, and C H Polman (One year changes in disability in multiple sclerosis: neurological examination compared with patient self report) in the April issue of JNNP (JNNP 2003;74:439-42).

Objective: To characterise the relation between one year changes in neurologist rating of neurological exam abnormalities as measured by the EDSS and changes in patient perceived disability as measured by the GNDS in patients with MS

Methods: 250 patients with MS were recruited at an outpatient clinic. Disability at baseline and one year follow up was assessed using the EDSS and GNDS. Correlations between change in EDSS, GNDS-sum score, functional systems, and GNDS subcategories were studied as well as the significance of changes in EDSS associated with changes in perceived disability.

Results: The correlation between one year changes in EDSS $v$ GNDS was substantially lower $(0.19)$ than cross-sectional correlations between EDSS and GNDS, either at baseline (0.62) or at follow up (0.77). Notably, changes in functional system scores that are based on neurological examination are poorly or not at all correlated with changes in disability as perceived by the patient. Analysing the impact of a significant worsening in EDSS score we found that this was associated with significant worsening, insignificant change, and significant improvement in the patients' perceived disability in $45 \%, 39 \%$, and $15 \%$ of patients, respectively.

Conclusion: Patients' perception of change in disability differs not only quantitatively but also qualitatively from that of an examining physician. There are true differences in change as perceived by the patient and measured by the physician and changes in many dimensions of disability are relevant to the patient and have no measurable impact on the EDSS.

The authors of the short report entitled Paraneoplastic ophthalmoplegia and subacute motor axonal neuropathy associated with anti-GQlb antibodies in a patient with malignant melanoma, published in the April issue 2003 of JNNP (2003;74:507-9), were listed in the incorrect order. The author order should read as follows: L Kloos, C W Ang, W Kruit, G Stoter, and P Sillevis. 